\title{
Infrared Absorption Enhancement Using Periodic Inverse Nanopyramids in Crystalline-Silicon Bottom Cells for Application in Tandem Devices
}

\author{
Arsalan Razzaq ${ }^{\circledR}$, Valérie Depauw, Hariharsudan Sivaramakrishnan Radhakrishnan, Jinyoun Cho, Ivan Gordon, \\ Jozef Szlufcik, Yaser Abdulraheem, and Jef Poortmans
}

\begin{abstract}
Carefully tailored periodic nanostructures on the light wavelength scale, such as diffraction gratings, benefit from wave optics for efficiently trapping the weakly absorbing infrared photons in crystalline-silicon (c-Si) absorbers. In contrast with the conventional random pyramid texture, diffraction gratings can be designed to target specific wavelength ranges by the selection of the grating pitch. Absorption enhancement at infrared wavelengths in a silicon solar cell is especially desired when it operates below a perovskite top cell in a tandem device. In this article, inverse nanopyramid gratings of $800 \mathrm{~nm}$ pitch are proposed as an alternative front-surface texture to random pyramids in silicon heterojunction devices with interdigitated back contacts that are to be used as bottom cells in four-terminal perovskite/c-Si tandem devices. By doing so, we report a short-circuit current density gain of $0.53 \mathrm{~mA} / \mathrm{cm}^{2}$ with respect to the random pyramid texturing for the bottom c-Si cell. The rationale to substitute random pyramids by inverse nanopyramid gratings is, however, not justified in single-junction operation despite achieving the power conversion efficiency of $22.3 \%$ since the degraded optical performance at shorter wavelengths offsets the absorption enhancement at longer wavelengths, resulting in similar levels of short-circuit current densities for both texture types.
\end{abstract}

Index Terms-Heterojunction, interdigitated and back contact (IBC), light trapping, nanoimprint lithography (NIL), nanophotonics, solar cells, tandem cells.

Manuscript received November 22, 2019; revised January 30, 2020; accepted February 3, 2020. This work was supported by the Kuwait Foundation for the Advancement of Sciences under Project no. CN18-15EE-01. (Corresponding author: Arsalan Razzaq.)

Arsalan Razzaq is with the Departement Elektrotechniek (ESAT), Katholieke Universiteit Leuven, 3001 Leuven, Belgium, and also with IMEC (Partner in EnergyVille), 3001 Leuven, Belgium (e-mail: arsalan.razzaq@imec.be).

Valérie Depauw, Hariharsudan Sivaramakrishnan Radhakrishnan, Ivan Gordon, and Jozef Szlufcik are with theIMEC (Partner in EnergyVille), 3001 Leuven, Belgium (e-mail: valerie.depauw@imec.be; hariharsudan.sivaramakrishnan@imec.be; ivan.gordon@imec.be; jozef.szlufcik@ imec.be).

Jinyoun Cho is with the Departement Elektrotechniek (ESAT), Katholieke Universiteit Leuven, 3001 Leuven, Belgium (e-mail: jinyoun.cho.ext@imec.be).

Yaser Abdulraheem is with the Department of Electrical Engineering, Kuwait University, Safat 13060, Kuwait (e-mail: yaser.abdulraheem@ku.edu.kw).

Jef Poortmans is with the Departement Elektrotechniek (ESAT), Katholieke Universiteit Leuven, 3001 Leuven, Belgium, and with IMEC (Partner in EnergyVille), 3001 Leuven, Belgium, and also with Universiteit Hasselt, 3500 Hasselt, Belgium (e-mail: jef.poortmans@imec.be).

Color versions of one or more of the figures in this article are available online at http://ieeexplore.ieee.org.

Digital Object Identifier 10.1109/JPHOTOV.2020.2972324

\section{INTRODUCTION}

$\mathbf{S}$ IGNIFICANT advancement in the silicon photovoltaics (PV) technology over the past decades has drastically reduced the price of silicon solar cells and modules. With solar panels becoming increasingly more affordable, further reduction in the levelized cost of electricity is predominantly now constrained by the area-dependent installation expenses [1]. Examples of these expenses, labeled as the balance-of-system costs, include the cost of wiring, mounting structures, inverters, batteries, and other components constituting a PV system. Since the balance-of-system costs scale-up with the area, they can potentially be lowered by raising the cell and module-level power conversion efficiencies (PCEs) so that more power can be generated per unit area.

Crystalline-silicon (c-Si) solar cells have already attained remarkable conversion efficiencies beyond 26\% [2]-[4], thereby closing the gap with the intrinsic limit of $29.4 \%$ [5]. However, the practical limit for c-Si cells is estimated to be only around $27 \%$ once the resistive losses are taken into consideration [3]. Therefore, room for further efficiency improvement with single-junction ( $\mathrm{S}-\mathrm{J}) \mathrm{c}$-Si cells is marginal and calls for the application of more sophisticated cell concepts to surpass the fundamental S-J c-Si efficiency barrier [6]. One of the reasons for the relatively low fundamental efficiency limit of c-Si cells is the nonoptimal utilization of excess photon energy. Being referred to as thermalization losses, the extra energy of ultraviolet wavelength photons above the bandgap of $\mathrm{c}-\mathrm{Si}$ is wasted as the photogenerated carriers relax to the conduction and valence band edges by emitting phonons. Mitigating thermalization losses is possible, for instance, by employing the multijunction approach in which several absorber materials of complementary bandgaps are stacked on top of one another such that the higher energy photons are absorbed in wider bandgap materials, while the narrower bandgap absorbers only receive the lesser energy photons. This way, the total efficiency can be raised above that of individual subcells that make up the multijunction cell structure. Since most progress in PV research for terrestrial applications is driven by cost-savings, the double junction or the tandem configuration represents the simplest and most cost-effective multijunction cell structure with the potential of reaching PCE values as high as $46 \%$ [7] under the standard AM $1.5 \mathrm{G}$ solar spectrum and intensity. 
In combination with a c-Si bottom cell, organic-inorganic hybrid perovskites have emerged in recent years as promising top-cell candidates for tandem devices [7]-[15]. Besides, the low processing cost of perovskites using solution-based [16], [17] or vapor deposition [18] methods, perovskites exhibit a steep absorption edge [19] and their bandgap can be tuned over a wide range [20], [22], making them attractive top-cell absorbers. Perovskite/c-Si tandem devices are normally realized in two-terminal (2-T) and four-terminal (4-T) configurations with each design offering certain advantages over the other. The 2-T design involves the deposition of perovskite cell layers on an underlying c-Si cell so that both subcells are electrically connected in series. The 2-T design is attractive since additional circuitry at the system level is not required while keeping the parasitic absorption losses minimal by only utilizing a single transparent electrode. However, the current-matching requirement of the configuration [23] demands tight control over the bandgap and the thickness of the top-cell absorber layer. Another challenge with the 2-T design is to conformally deposit the perovskite cell layers on a textured $\mathrm{c}-\mathrm{Si}$ bottom cell without damaging the functional layers underneath [24]. On the other hand, in the 4-T case, individual subcells can be optimized and fabricated separately and later coupled optically by mechanical stacking. The configuration, therefore, offers more freedom in the choice of bottom c-Si cell architecture, specifically now tailored to operate under the infrared (IR) part of the AM 1.5G spectrum. Perhaps the biggest advantage of the 4-T configuration over its 2-T rival is the absence of current-matching requirement between the subcells. It implies that a 4-T device efficiency is less sensitive to spectral composition variations, translating into a higher annual energy yield [66], [67], to compensate for the additional circuitry cost at the system level.

In order to maximize the open-circuit voltage $\left(V_{\mathrm{OC}}\right)$ of the narrower bandgap c-Si subcell, cell technologies incorporating passivating contacts [25], [26] could be used to reach the unprecedented levels of $V_{\mathrm{OC}}$ by significantly curtailing carrier recombination losses at the contacts [27]-[32]. Amongst the numerous materials reported to implement passivating contacts, the highest voltages to date for S-J c-Si cells were achieved by deploying hydrogenated amorphous silicon (a-Si:H) thin-film stacks [33], [34]. In this silicon heterojunction (SHJ) cell technology, excellent surface passivation is enabled by depositing intrinsic and in situ doped a-Si:H thin-film stacks that saturate the dangling bonds at the surfaces and provide band bending at the c-Si surface for the carrier selectivity [35], [36]. Moreover, it is also possible to eliminate grid-shading losses by implementing both terminals of the device on the rear side in aninterdigitated and back-contacted (IBC) cell structure [37]. The merger of SHJ and IBC cell concepts, i.e., SHJ-IBC, led to the record $26.7 \%$ cell efficiency for S-J c-Si devices [2]. From the perspective of 4-T tandem devices, the SHJ-IBC architecture is particularly well suited for bottom-cell usage as both contacts are situated on the rear side, which reduces the number of required transparent electrodes by one. Another optical advantage of the structure is that the passivating a-Si:H stacks are transparent to the incoming IR photons, thereby allowing the adaptation of their thickness for optimal electrical performance without contributing to additional parasitic absorption losses. Unlike diffused junction cells, free-carrier absorption losses are also minimized in this cell structure. Finally, the SHJ-IBC architecture can be used in conjunction with the advanced light management strategies to boost the IR absorption in these devices.

Advanced light management strategies nowadays include substituting the industry-standard random pyramid (RP) texture by diffraction gratings [38]-[46], [58], black-silicon [47]-[49], or other nanoscale textures [50]-[56] in solar devices. Amongst many nanoscale texturing alternatives, periodic wavelengthscale textures, such as gratings, profit from diffraction to enhance absorption at wavelengths corresponding to the grating pitch [43], [57]. In the case of RP texture, trapping efficiency of in-coupled photons within the absorber is dependent on the incidence angle as described by geometrical optics. However, this angular dependence can be circumvented by trapping light instead of in the wave optics regime. For the wave-optical case, superior optical performance with respect to RP texture at desired wavelengths is attributed to the excitation of waveguide modes in the photoactive layer, which are controlled by the grating pitch [59]. The introduction of diffraction gratings on the rear side of the c-Si bottom cell in a 2-T GaInP/GaAs//c-Si triple-junction device led to an impressive $33.3 \%$ PCE [60]. This work by Cariou et al. [60] demonstrates a short-circuit current density $\left(J_{\mathrm{SC}}\right)$ gain in the c-Si subcell of $1.1 \mathrm{~mA} / \mathrm{cm}^{2}$ compared with the flat reference. In a 4-T configuration, where there are no constraints on the geometry of the front-side texture, it remains to be ascertained if the presence of diffraction gratings on the front side of a c-Si bottom cell is optically more favorable than its RP-textured analog, which is the main goal of this article.

In this article, we propose the integration of inverse nanopyramid (INP) gratings as the front-surface texture in SHJ-IBC cell structure for application in 4-T perovskite/c-Si tandem devices. We demonstrate increased IR absorption in c-Si devices by frontside nanopatterning as opposed to the standard RP texturing, thus providing an additional route to enable higher bottom-cell efficiencies.

\section{EXPERIMENTAL DETAILS}

\section{A. Nanoimprint lithography (NIL) Process}

$\mathrm{SiO}_{\mathrm{X}}$ was deposited by plasma-enhanced chemical vapor deposition (PECVD) at $225^{\circ} \mathrm{C}$ on both sides of the c-Si substrate ( $n$-type float zone, $200 \mu \mathrm{m}$ thick, and $3.0 \Omega \cdot \mathrm{cm}$ base resistivity) to serve as metal-contamination protection on the rear and lithography mask on the front. Resist (mr-I 7010R from microresist technology) was then spun on to the side to be patterned. Spin coating was followed by pattern transfer from the soft stamp (silicone elastomer Sylgard 184 from Dow) to the resist by thermal imprinting in a hydraulic press at $130{ }^{\circ} \mathrm{C}$. $\mathrm{Next}, \mathrm{SiO}_{\mathrm{X}}$ on the front side was patterned by consecutive $\mathrm{O}_{2}$ and $\mathrm{CF}_{4}$ reactive ion etching (RIE) steps. The INP pattern was formed by wet chemical anisotropic etching in TMAH: $\mathrm{H}_{2} \mathrm{O}=$ 2: 3 solution with a commercial surfactant at $80 \pm 2{ }^{\circ} \mathrm{C}$. In the last step, $\mathrm{SiO}_{\mathrm{X}}$ was removed by a short dip in $\mathrm{HF}$ : $\mathrm{HCl}: \mathrm{H}_{2} \mathrm{O}=$ 1: 1: 20 solution. The NIL process is depicted in Appendix A 
and reported in detail in previous works with adaptation in RIE steps [44], [45].

\section{B. Random Pyramid (RP) Texturing}

Reference single-side RP-textured samples were prepared by protecting one side by depositing $\mathrm{PECVD} \mathrm{SiO}_{\mathrm{X}} / \mathrm{SiN}_{\mathrm{X}}$ as a hard mask. Texturing was performed in TMAH: $\mathrm{H}_{2} \mathrm{O}=1: 20$ solution with commercial surfactant at $80{ }^{\circ} \mathrm{C}$. The hard mask, after texturing, was then dissolved in $\mathrm{HF}: \mathrm{HCl}: \mathrm{H}_{2} \mathrm{O}=1: 1: 20$ solution.

\section{Fabrication of Silicon Heterojunction Devices With Interdigitated Back Contacts (SHJ-IBC)}

The flat rear of the single-side-textured wafers was coated with PECVD (i/p) a-Si:H and PECVD $\mathrm{SiO}_{\mathrm{X}}$ hard mask before photolithography, dry etching, and lift-off steps that define the interdigitated pattern. Passivation of the front surface by (i) a$\mathrm{Si}: \mathrm{H}$ and $\mathrm{SiN}_{\mathrm{X}}$ antireflection coating (ARC) was followed by sputtering indium-doped tin oxide (ITO) and evaporating $\mathrm{Cu}$ on the rear to form the electrodes. Lithography and wet etching were again performed to achieve electrical isolation between the contacts. For more details on the rear-side patterning, the reader can refer to our previous publications on the subject [63]-[65].

\section{Cell Characterization}

Current density $(J)$-voltage $(V)$ measurements of solar cells were performed under standard testing conditions (AM 1.5G $1000 \mathrm{~W} / \mathrm{m}^{2}$ solar spectrum and $25^{\circ} \mathrm{C}$ temperature) using a WACOM solar simulator. The WACOM simulator was calibrated before measurements using an externally certified SHJ-IBC cell. Reflectance (R) and external quantum efficiency (EQE) measurements were made using the LOANA solar cell analysis system from PV tools $\mathrm{GmbH}$, with an illumination spot size of $1.5 \times 1.5 \mathrm{~cm}^{2}$. Samples were prepared for transmission electron microscopy (TEM) measurements as detailed in Appendix B.

\section{RESULTS AND DISCUSSION}

\section{A. Investigated Pyramid Textures}

Scanning electron microscopy (SEM) images of the investigated textures are presented in Fig. 1. The NIL process, briefly described in Section II, was applied to realize periodic INP gratings with an $800 \mathrm{~nm}$ pitch, in order to target the near-IR part of the incoming spectrum of light. Our nanopatterned inverse pyramids feature a square base of a size that is defined by the area filling fraction (AFF) and the grating pitch. The AFF is the ratio of the area with INPs to the total area and is a measure of the width of flat regions adjoining the INPs. Reaching an ideal AFF of unity is theoretically possible with square base shape patterns but given the variation in the NIL process due to substrate roughness, presence of localized defects, and soft-stamp deformations [61], obtaining near-ideal AFF is experimentally very challenging. In our case, we reached AFFs close to 0.85, which is equivalent to a pyramid base width of $\sim 735 \mathrm{~nm}$. More importantly, the nanopyramid dimensions are comparable with
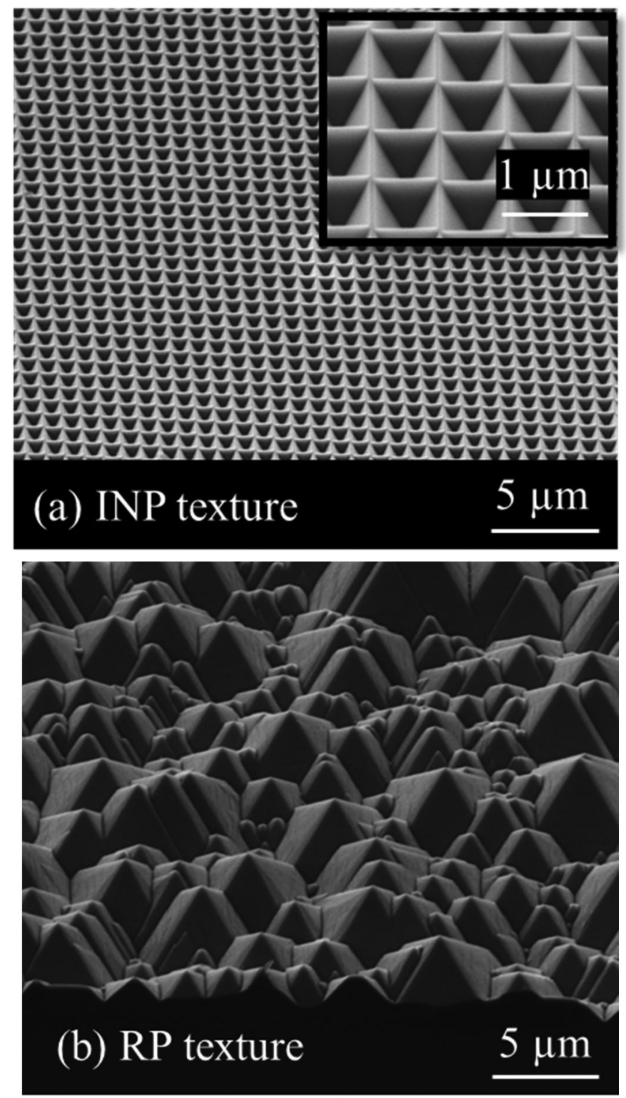

Fig. 1. SEM images of the textures that were integrated into SHJ-IBC devices. (a) Periodic INP gratings of $800 \mathrm{~nm}$ pitch were fabricated by NIL. (b) RP texture formed by alkaline etching serves as the reference.

the wavelength of in-coupled photons so that the wave effects become relevant in the wavelength range of interest. On the contrary, the RP texture comprises much larger pyramids of base width size $\sim 2-5 \mu \mathrm{m}$ and provides path length improvement for in-coupled photons by refracting them at large angles within the absorber.

\section{B. Adapting the Antireflection Coating Layer Thickness}

Both investigated pyramid textures were incorporated on the front side of SHJ-IBC devices, as shown schematically in Fig. 2, with the rear side kept flat for ease in the rear-side patterning steps during cell processing. Process details on the fabrication of these devices are briefly described in Section II and covered more extensively in our previous publications [63]-[65]. With the exception of the front-surface texture and ARC layer thickness, both device types were identical in structure and processed together. The thickness of the $\mathrm{SiN}_{\mathrm{X}} \mathrm{ARC}$ was adapted for each texture to yield low reflection losses in the 500-1200 nm wavelength interval, which corresponds to the transmittance spectrum of the perovskite top cell.

We considered spectrum-weighted reflectance (SWR) as the figure of merit in deciding the optimal $\mathrm{SiN}_{\mathrm{X}}$ thickness values, which were controlled experimentally by varying deposition times. The optimization, aimed to minimize the SWR in the stated wavelength range for each texture, was carried out by 
(i) $\mathrm{a}-\mathrm{Si}: \mathrm{H} / \mathrm{SiN}_{\mathrm{X}}$

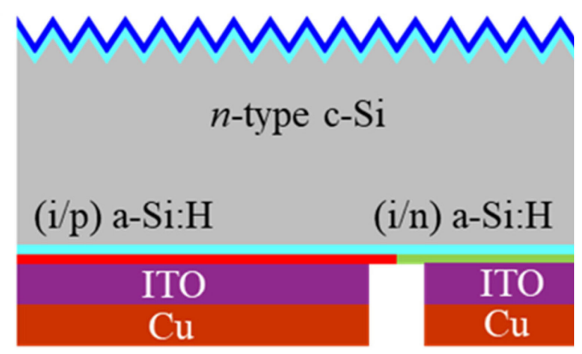

(a)

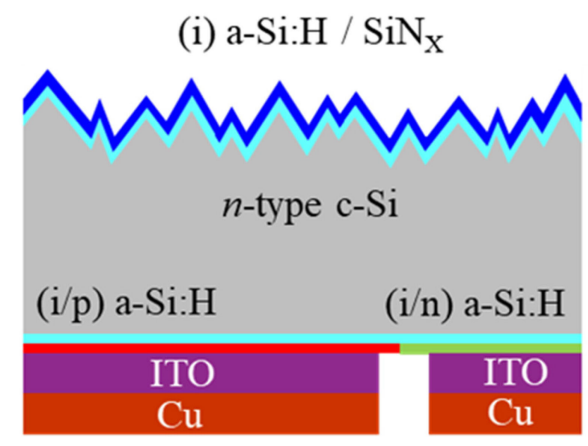

(b)

Fig. 2. Schematic structures of SHJ-IBC devices are shown for both (a) INP and (b) RP textures.

depositing $\mathrm{SiN}_{\mathrm{X}}$ on the textured c-Si samples using different deposition times and measuring the reflectance spectra of these samples, followed by computing the SWR. The condition leading to the lowest SWR for each texture was then selected. The SWR takes the incident photon flux into account and is given by

$$
\mathrm{SWR}=1-\frac{\int_{\lambda_{1}}^{\lambda_{2}}(1-R) \cdot \varphi_{\mathrm{AM} 1.5 \mathrm{G}} \cdot \partial \lambda}{\int_{\lambda_{1}}^{\lambda_{2}} \varphi_{\mathrm{AM} 1.5 \mathrm{G}} \cdot \partial \lambda}
$$

where $\lambda_{1}-\lambda_{2}$ is the chosen wavelength interval, $R$ is the wavelength-dependent reflection coefficient, and $\varphi_{\mathrm{AM}} 1.5 \mathrm{G}$ is the AM $1.5 \mathrm{G}$ photon flux.

In the wavelength interval of 500-1200 $\mathrm{nm}$ in which the perovskite top cell was assumed to behave as a sharp bandpass filter, the minima in SWR values of $5.3 \%$ for the INP texture and $6.0 \%$ for the RP texture was found during optimization and the corresponding $\mathrm{SiN}_{\mathrm{X}}$ thicknesses were determined from TEM cross-sectional images, as shown in Fig. 3. The optimal deposition time for the INP texture was found to be shorter, and consequently, the average thickness of $\mathrm{SiN}_{\mathrm{X}}$ on the INPs was lower (between 40 and $75 \mathrm{~nm}$ ) in contrast with that on the RP texture (uniformly $\sim 75 \mathrm{~nm}$ ). This difference in the optimal thickness could be explained by the choice of grating pitch for the INP texture, meant to target near-IR wavelengths for tandem operation, consequently leading to higher $R$ losses in the intermediate wavelength range $(500-800 \mathrm{~nm})$, which is tackled by implementing a slightly thinner ARC layer to target this wavelength range. Moreover, it can be observed from TEM images that unlike the case of the $\mathrm{RP}$ texture, $\mathrm{SiN}_{\mathrm{X}}$ is nonconformal and

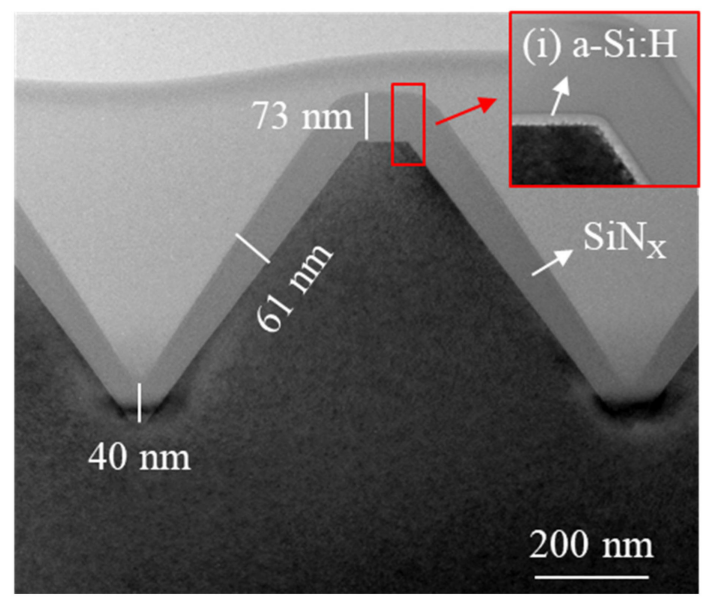

(a)

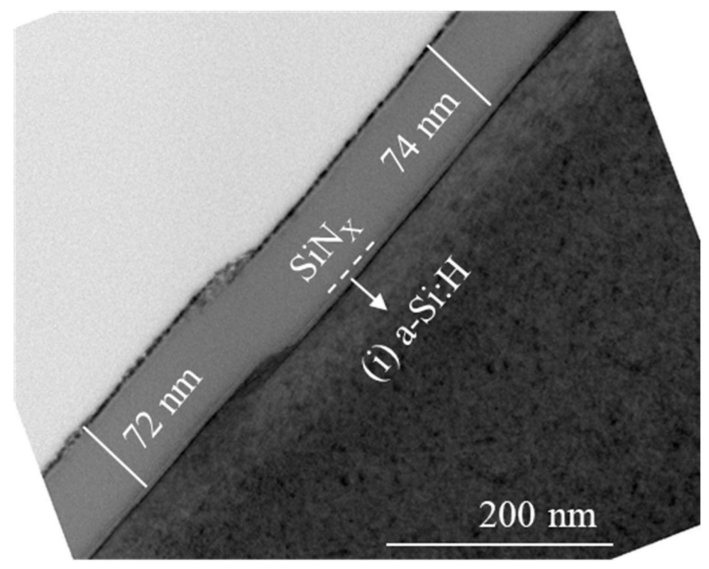

(b)

Fig. 3. TEM cross-sectional images showing $\mathrm{SiN}_{\mathrm{X}}$ thickness on pyramid facets for (a) INP and (b) RP textures. $\mathrm{SiN}_{\mathrm{X}} \mathrm{ARC}$ is nonconformal and thinner on the facets of the nanopyramid pattern.

shows thickness variation along the nanopyramid topography, varying uniformly between $\sim 75 \mathrm{~nm}$ on the plateaus and $\sim 40 \mathrm{~nm}$ in the valleys of the nanopyramids. The nonconformality arises due to the nanopyramid topography, which consists of pyramidal "dents" as opposed to outward projecting pyramids in the case of RPs. This variation in the deposition rate can be explained by the diminished influx of reactant species to the constrained pyramid valleys or conversely because of the slower desorption of reactant products. Therefore, lowering the surface temperature and deposition power may lead to more conformally deposited $\mathrm{SiN}_{\mathrm{X}}$ films on nanopyramid-textured surfaces [68] but require a thoughtful optimization as other properties of the film may be affected.

\section{Solar Cell Results in Single-Junction and Tandem Operations}

$J-V$ parameters of SHJ-IBC solar cells, featuring an ARC optimized to minimize $R$ losses in the 500-1200 nm wavelength interval and an active area of $3.97 \mathrm{~cm}^{2}$, are given in Fig. 4 and Table I for S-J operation under the standard AM 1.5G 

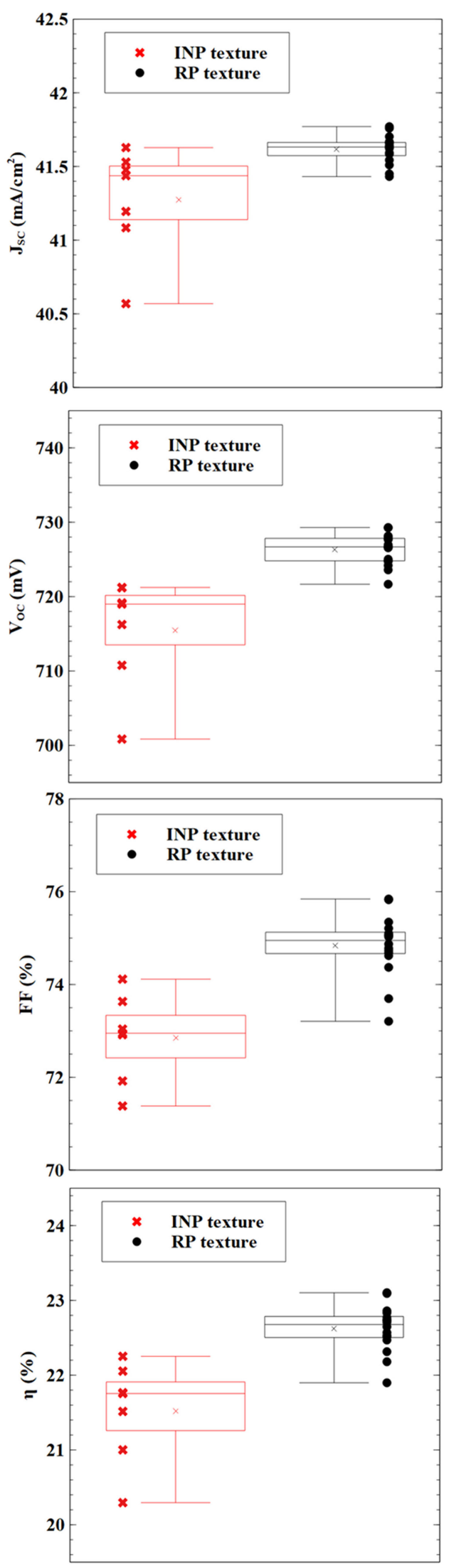

Fig. 4. Comparison of illuminated $J-V$ results of SHJ-IBC devices with an area of $3.97 \mathrm{~cm}^{2}$ in S-J operation under the AM $1.5 \mathrm{G}$ spectrum with INP and RP surface textures. RP-textured cells reached higher efficiencies.
TABLE I

SUMMARY OF ILLUMINATED $J-V$ RESULTS OF SHJ-IBC DEVICES IN S-J OPERATION FOR BOTH INVESTIGATED SURFACE TEXTURES

\begin{tabular}{cccccc}
\hline Text. & & $\begin{array}{c}\mathbf{J}_{\mathbf{S C}} \\
\left(\mathbf{m A} / \mathbf{c m}^{2}\right)\end{array}$ & $\begin{array}{c}\mathbf{V}_{\mathbf{O C}} \\
(\mathbf{m V})\end{array}$ & $\begin{array}{c}\mathbf{F F} \\
\mathbf{( \% )}\end{array}$ & $\begin{array}{c}\boldsymbol{\eta} \\
(\mathbf{\%})\end{array}$ \\
\hline \multirow{2}{*}{ INP } & $\begin{array}{c}\text { Avg. } \\
\text { of 7 }\end{array}$ & 41.27 & 715 & 72.9 & 21.5 \\
& & \pm 0.36 & \pm 7 & \pm 0.9 & \pm 0.6 \\
RP & Avg. & 41.64 & 726 & 74.8 & 22.6 \\
& of 11 & \pm 0.08 & \pm 2 & \pm 0.7 & \pm 0.3 \\
& & & & & \\
INP & Best & 41.63 & 721 & 74.1 & 22.3 \\
RP & Best & 41.77 & 729 & 75.8 & 23.1 \\
& & & & & \\
\hline
\end{tabular}

illumination spectrum and testing conditions. As expected for S-J configuration, the INP patterned cells were outperformed by their RP-textured counterparts. Outstanding $V_{\mathrm{OC}}$ values above $700 \mathrm{mV}$ have been achieved for both texture types, proving that INP patterning of the surface does not significantly compromise the passivation quality [58]. The slightly lower $V_{\mathrm{OC}}$ and fill factor $(\mathrm{FF})$ values for the INP pattern were due to larger nonideal recombination $\left(J_{02}\right)$ losses, which we believe could be attributed to RIE-induced surface damage during NIL patterning (step 6 in Appendix A), and the nonconformal intrinsic (i) a-Si:H layer thickness. The latter exhibits a similar nonconformality in thickness as was observed in the case of PECVD $\mathrm{SiN}_{\mathrm{X}}$, which makes it thinner in the valleys than our required target thickness of $5 \mathrm{~nm}$ for adequate chemical passivation (see Appendix C). Thus, there is room for further improvement in the $V_{\mathrm{OC}}$ and $\mathrm{FF}$ of INP-textured cells by improving the conformality of the front side (i) a-Si:H passivation layer. Nevertheless, our best INP patterned device reached an excellent cell efficiency $(\eta)$ of $22.3 \%$ compared with $23.1 \%$ for the RPtextured reference in standalone operation under a full AM 1.5G spectrum.

In order to understand the relative optical performance of the two investigated textures in the different regions of the spectrum, we conducted $\mathrm{R}$ and EQE measurements on representative devices of both types of texture (see Fig. 5), which revealed that the escape losses for wavelengths between 950 and $1200 \mathrm{~nm}$ were much lower in the case of INP texture with an SWR of $13.6 \%$ as opposed to $18.7 \%$ for the RP texture, translating into a potential $J_{\mathrm{SC}}$ gain of $0.50 \mathrm{~mA} / \mathrm{cm}^{2}$ for this wavelength range. However, the actual $J_{\mathrm{SC}}$ gain from EQE results in the stated wavelength interval turns out to be $0.60 \mathrm{~mA} / \mathrm{cm}^{2}$, which points to additional optical advantage of INP patterning besides superior IR photon trapping. We postulate that the excitation of higher 


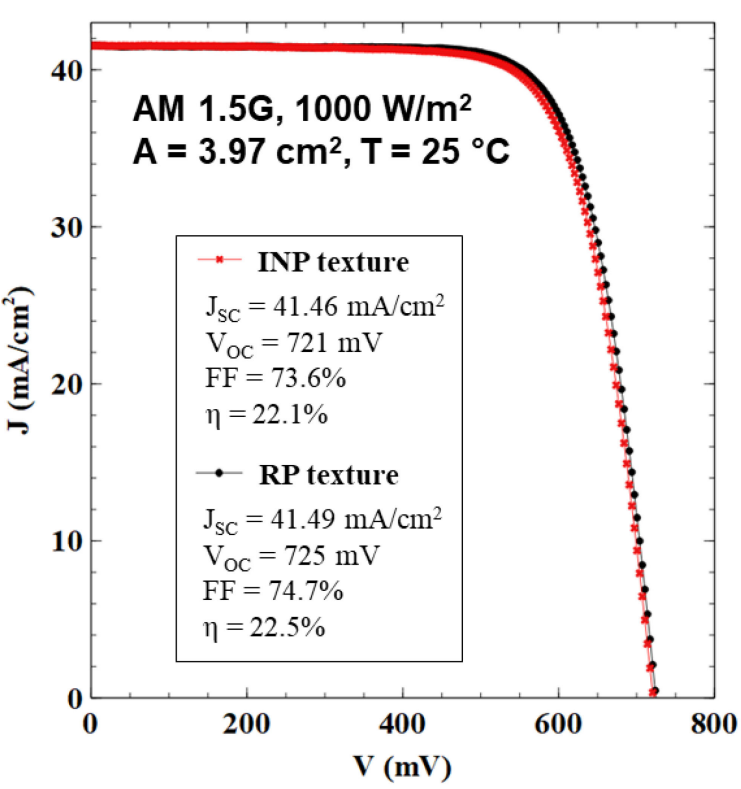

(a)

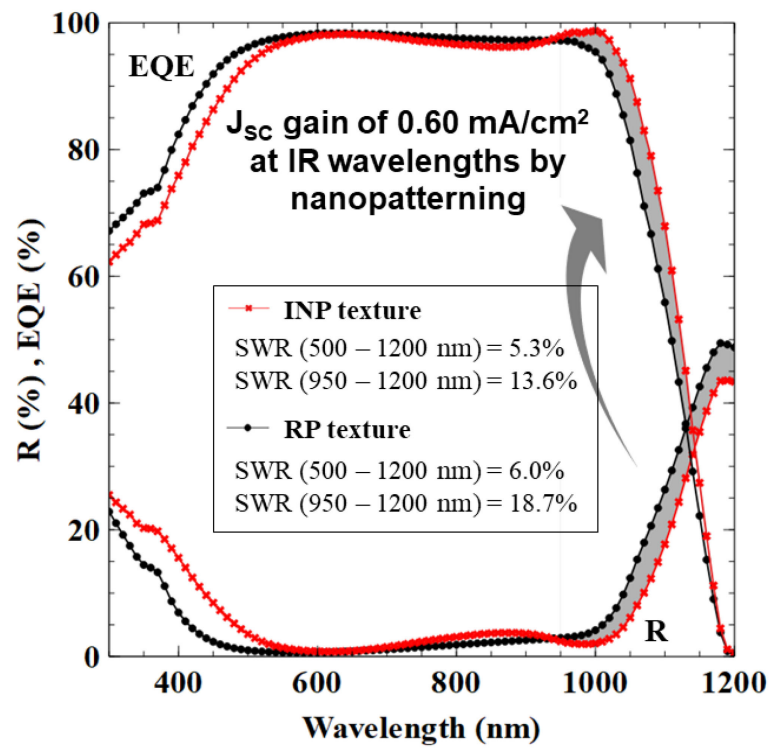

(b)

Fig. 5. (a) Representative SHJ-IBC devices were selected from each texture split for R and EQE comparison in S-J operation. (b) Nanopatterned SHJ-IBC device shows superior IR performance than its RP-textured analog.

order modes by the nanopyramid grating texture may yield lower parasitic absorption losses in the rear-side ITO and Cu contacts from the higher angle light in-coupling it provides. Nonetheless, further investigation is required to identify the reason behind the observed discrepancy of $0.10 \mathrm{~mA} / \mathrm{cm}^{2}$ between $\mathrm{R}$ and $\mathrm{EQE}$ measurements.

Although Fig. 5(b) also shows that the RP texture provides better light in-coupling at shorter wavelengths $(<550 \mathrm{~nm}) \mathrm{com}$ pared with an INP grating of $800 \mathrm{~nm}$ pitch, these wavelengths are not relevant in the context of a c-Si bottom cell in tandem configuration.
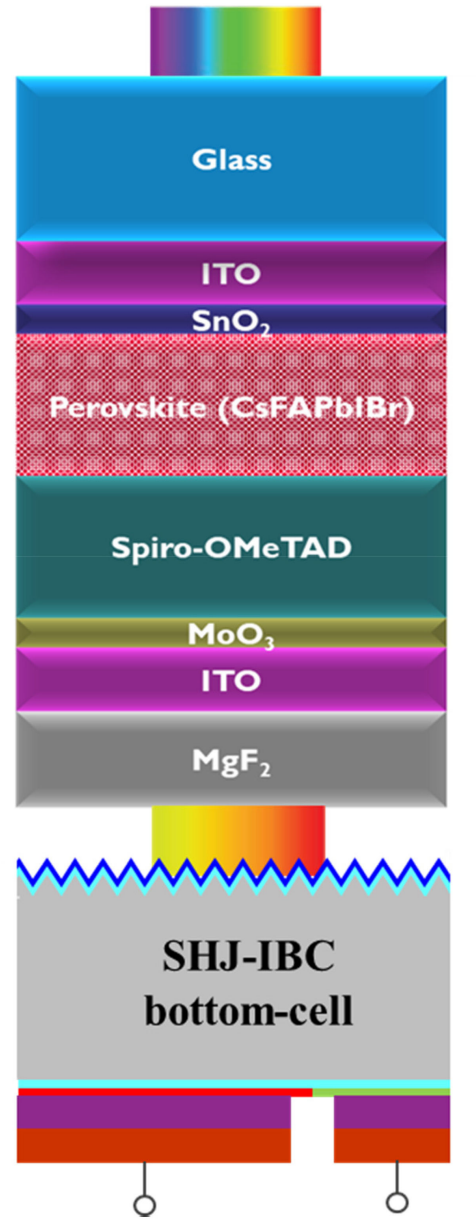

(a)

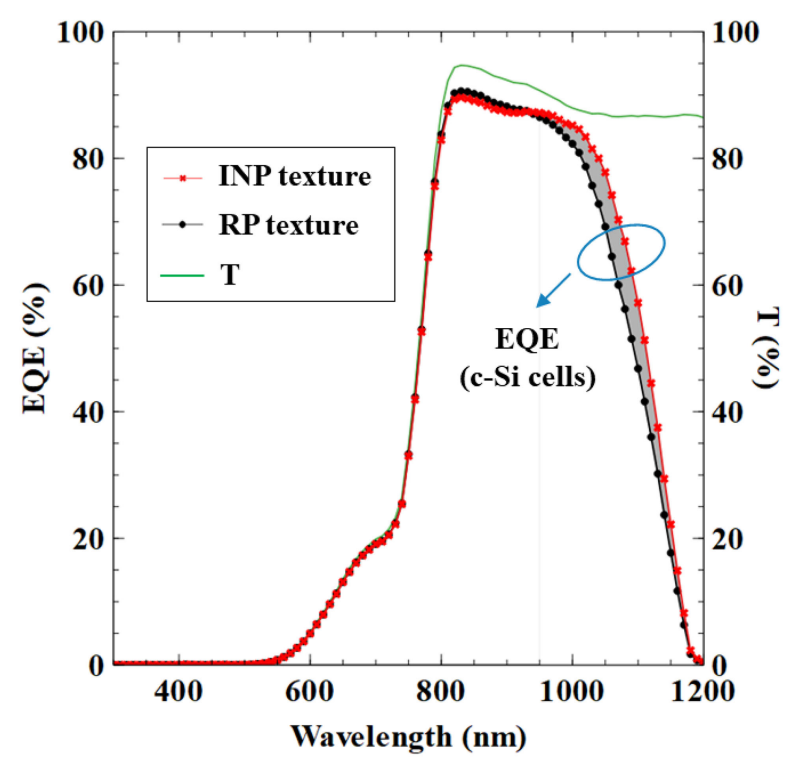

(b)

Fig. 6. (a) Schematic structure of a planar perovskite device without contacts that was placed on top of SHJ-IBC bottom cells for EQE and $J-V$ measurements in 4-T tandem operation. The transmitted spectrum reaches the bottom c-Si cell through an airgap. (b) EQE spectra of SHJ-IBC cells underneath a perovskite device precursor of transmittivity (T). The IR boost with nanopatterning was also observed in the 4-T tandem operation. 
TABLE II

ILLUMINATED $J-V$ RESULTS OF SHJ-IBC DEVICES IN TANDEM OPERATION

$\begin{array}{ccccc}\text { Text. Config. } & \begin{array}{c}\mathbf{J}_{\mathrm{SC}} \\ \left(\mathrm{mA} / \mathrm{cm}^{2}\right)\end{array} & \begin{array}{c}\mathrm{V}_{\mathrm{OC}} \\ (\mathrm{mV})\end{array} & \begin{array}{c}\mathrm{FF} \\ (\%)\end{array} & \begin{array}{c}\eta \\ (\%)\end{array}\end{array}$

$\begin{array}{cccccc}\text { INP } & \text { S-J } & 41.46 & 721 & 73.6 & 22.1 \\ \text { RP } & \text { S-J } & 41.49 & 725 & 74.7 & 22.5 \\ & & & & & \\ \text { INP } & \text { Tandem } & 17.86 & 692 & 76.8 & 9.5 \\ \text { RP } & \text { Tandem } & 17.33 & 693 & 77.9 & 9.3\end{array}$

$J-V$ parameters of both devices in S-J operation are also shown for reference.

The efficiency enhancement due to the INP texture in 4-T tandem configuration was then analyzed by measuring the illuminated $J-V$ and EQE characteristics of these SHJ-IBC cells under the filtered AM $1.5 \mathrm{G}$ spectrum, placing them beneath a planar perovskite top-cell precursor of $n-\mathrm{i}-\mathrm{p}$ structure that features all supporting layers except the contacts [see Fig. 6(a)]. In this 4-T configuration, an airgap was present in between the subcells. Since the perovskite absorber layer with an optical bandgap of $\sim 1.7 \mathrm{eV}$ is transparent to IR wavelengths, we can assume that the reflected IR light from the bottom cell would partially be parasitically absorbed in the supporting layers of the top cell and the remainder would escape the tandem structure from the front side. Shorter wavelengths below $550 \mathrm{~nm}$, on the other hand, do not reach the bottom cell as they are absorbed by the top cell during the first pass. Consequently, the contribution of light that is redirected to the top cell from the bottom cell was neglected and the scope of the study was limited to the adaptation of bottom-cell design in 4-T tandem configuration. The EQE results in Fig. 6(b) and illuminated $J-V$ results in Table II present that by using an INP texture, the $J_{\mathrm{SC}}$ and the $\eta$ of c-Si bottom cell are higher than when using an RP texture in tandem configuration. It needs to be emphasized that the $J_{\mathrm{SC}}$ increment of $0.53 \mathrm{~mA} / \mathrm{cm}^{2}$ with respect to the RP texture benchmark in tandem operation was obtained using an INP grating with nonideal AFF. Therefore, we suspect that the improvement could be enhanced by accomplishing even higher AFFs or by introducing double-side gratings [62].

\section{CONCLUSION}

In this article, we demonstrated increased IR absorption in SHJ-IBC by integrating INP gratings. The gratings were prepared by NIL and compared with state-of-the-art RPs as the front-side texture to trap IR photons, with a view to their application in c-Si bottom cells in a 4-T perovskite/c-Si tandem device. Our cell results show that by using a grating pitch of $800 \mathrm{~nm}$ and tweaking the ARC layer thickness, improvement in IR absorption is offset by inferior light in-coupling at shorter wavelengths and leads to an overall reduced performance compared with the RP texture in S-J operation under the AM $1.5 \mathrm{G}$ spectrum. However, the IR absorption enhancement with nanopatterning becomes relevant when considering bottom-cell design for 4-T tandem devices. In our case, replacing the RP texture by INP gratings provided a short-circuit current density gain of $0.53 \mathrm{~mA} / \mathrm{cm}^{2}$ and elevated the bottom-cell efficiency.

\section{APPENDIX A}

SCHEMATIC PROCESS Flow OF THE THERMAL NIL PROCESS TO FABRICATE INP GRATINGS

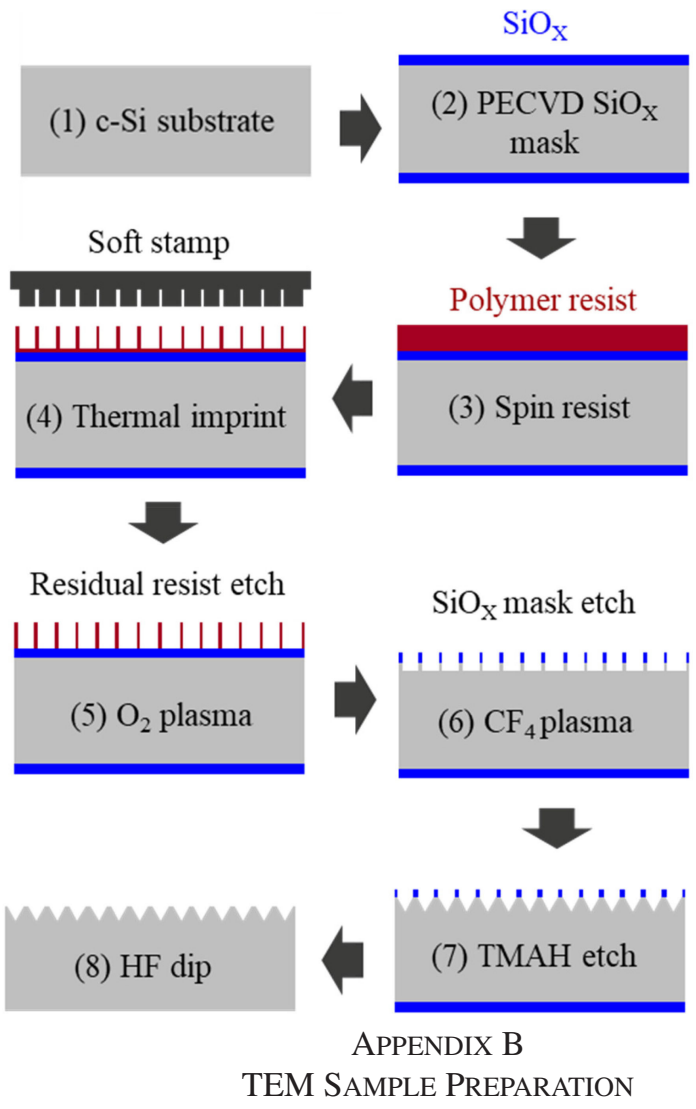

TEM sample preparation consists of the following steps.

1) Spin-on Carbon $(\mathrm{SoC})$ is deposited on the die before the sample enters the focused ion beam (FIB). At the FIB, Pt is deposited on the region of interest (and on top of SoC) by ion beam deposition. The FIB uses Ga ions to mill around the region of interest (now covered by SoC $+\mathrm{Pt}$ ) so that a cross section is extracted from the sample.

2) The extracted cross section is lifted away from the die and attached to a $\mathrm{Cu}$ grid.

3) The extracted cross section is thick $(\sim 3 \mu \mathrm{m})$ and is further thinned using also the $\mathrm{Ga}$ ion beam until the cross section is $<100 \mathrm{~nm}$ thick. In this step, both the back and front sides of the cross section are thinned an equal number of times until a thickness of $<100 \mathrm{~nm}$ is achieved. This is carried out using $\mathrm{Ga}$ ions at $5 \mathrm{kV}$. The cross section is thinned by moving the Ga ion beam downward from the top to the bottom of the sample. 


\section{APPENDIX C \\ Loss IN CHEMICAL PASSIVATION IN THE VALLEYS OF NANOPYRAMIDS}

\section{(i) a-Si:H layer thickness at the} nanopyramid plateaus

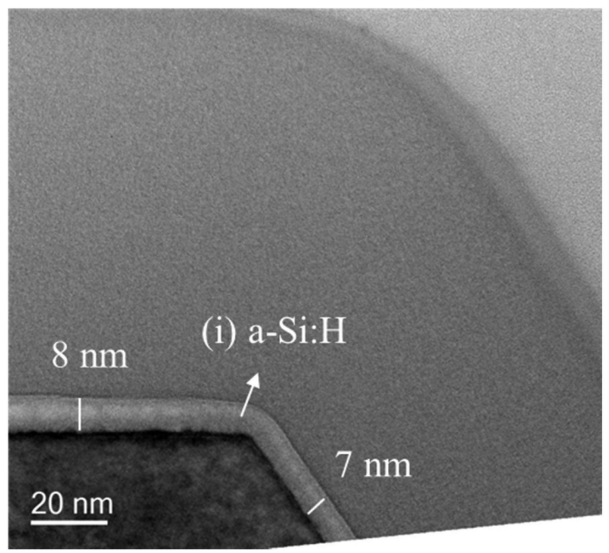

\section{(i) a-Si:H layer has non-optimal thickness} within the nanopyramid valleys

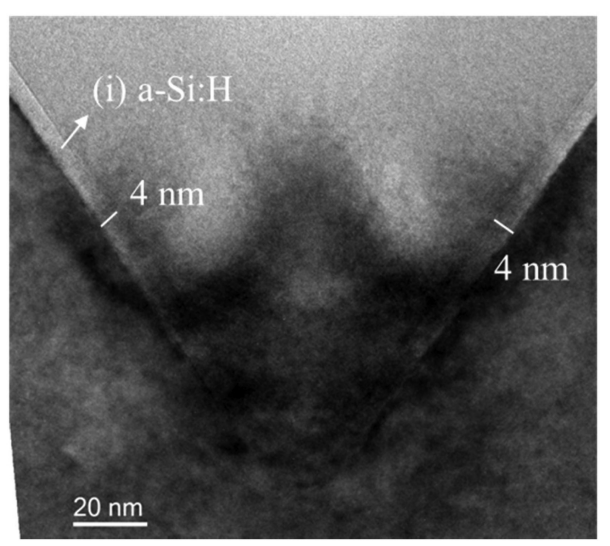

ACKNOWLEDGMENT

The authors would like to thank the partners of IMEC's industrial affiliation program for Silicon PV (IIAP-PV). IMEC is a partner in EnergyVille, ${ }^{1}$ a collaboration between the Flemish research partners KU Leuven, VITO, IMEC, and Universiteit Hasselt in the field of sustainable energy and intelligent energy systems. Optimization loops relating to $\mathrm{SiN}_{\mathrm{X}}$ thickness on nanopyramid-textured surfaces were carried out by T. Plancke. TEM inspection was performed by the Materials Components Analysis group of IMEC. Perovskite filters for $J-V$ and EQE measurements in tandem configuration were prepared by J. Sala and M. Jaysankar.

\section{REFERENCES}

[1] R. Fu, D. J. Feldman, and R. M. Margolis, "U.S. Solar photovoltaic system cost benchmark: Q1 2018," Nat. Renewable Energy Lab., NREL/TP6A20-72399, 2018.

[2] K. Yamamoto, K. Yoshikawa, H. Uzu, and D. Adachi, "High-efficiency heterojunction crystalline Si solar cells," Jpn. J. Appl. Phys., vol. 57, 2018, Art. no. 08RB20.

${ }^{1}$ [Online]. Available: www.energyville.be
[3] K. Yoshikawa et al., "Silicon heterojunction solar cell with interdigitated back contacts for a photoconversion efficiency over 26\%," Nature Energy, vol. 2, no. 5, 2017, Art. no. 17032.

[4] F. Haase et al., "Laser contact openings for local poly-Si-metal contacts enabling 26.1\%-efficient POLO-IBC solar cells," Sol. Energy Mater. Sol. Cells, vol. 186, pp. 184-193, 2018.

[5] A. Richter, M. Hermle, and S. W. Glunz, "Reassessment of the limiting efficiency for crystalline silicon solar cells," IEEE J. Photovolt., vol. 3, no. 4, pp. 1184-1191, Oct. 2013.

[6] W. Shockley and H. J. Queisser, "Detailed balance limit of efficiency of p-n junction solar cells," J. Appl. Phys., vol. 32, no. 3, pp. 510-519, 1961.

[7] T. Leijtens, K. A. Bush, R. Prasanna, and M. D. Mcgehee, "Opportunities and challenges for tandem solar cells using metal halide perovskite semiconductors," Nature Energy, vol. 3, no. 10, pp. 828-838, 2018.

[8] G. E. Eperon, M. T. Hörantner, and H. J. Snaith, "Metal halide perovskite tandem and multiple-junction photovoltaics," Nature Rev. Chem., vol. 1, no. 12, 2017, Art. no. 0095.

[9] J. Werner, B. Niesen, and C. Ballif, "Perovskite/silicon tandem solar cells: Marriage of convenience or true love story?-An overview," Adv. Mater Interfaces, vol. 5, no. 1, 2017, Art. no. 1700731.

[10] F. Fu et al., "High-efficiency inverted semi-transparent planar perovskite solar cells in substrate configuration," Nature Energy, vol. 2, no. 1, 2016, Art. no. 16190.

[11] T. Duong et al., "Rubidium multication perovskite with optimized bandgap for perovskite-silicon tandem with over $26 \%$ efficiency," Adv. Energy Mater, vol. 7, no. 14, 2017, Art. no. 1700228.

[12] J. Werner et al., "Efficient near-infrared-transparent perovskite solar cells enabling direct comparison of 4-terminal and monolithic perovskite/silicon tandem cells," ACS Energy Lett., vol. 1, no. 2, pp. 474-480, 2016.

[13] K. A. Bush et al., "23.6\%-efficient monolithic perovskite/silicon tandem solar cells with improved stability," Nature Energy, vol. 2, no. 4, 2017, Art. no. 17009

[14] M. Jaysankar et al., "Perovskite-silicon tandem solar modules with optimised light harvesting," Energy Environ. Sci., vol. 11, no. 6, pp. 1489-1498, 2018.

[15] U. W. Paetzold et al., "Scalable perovskite/CIGS thin-film solar module with power conversion efficiency of $17.8 \%$," J. Mater. Chem. A, vol. 5, no. 20, pp. 9897-9906, 2017

[16] M. Saliba et al., "Incorporation of rubidium cations into perovskite solar cells improves photovoltaic performance," Science, vol. 354, no. 6309, pp. 206-209, 2016.

[17] S. T. Williams, A. Rajagopal, C.-C. Chueh, and A. K.-Y. Jen, "Current challenges and prospective research for upscaling hybrid perovskite photovoltaics," J. Phys. Chem. Lett., vol. 7, no. 5, pp. 811-819, 2016.

[18] M. R. Leyden, Y. Jiang, and Y. Qi, "Chemical vapor deposition grown formamidinium perovskite solar modules with high steady state power and thermal stability," J. Mater. Chem. A, vol. 4, no. 34, pp. 13125-13132, 2016.

[19] S. D. Wolf et al., "Organometallic halide perovskites: Sharp optical absorption edge and its relation to photovoltaic performance," J. Phys. Chem. Lett., vol. 5, no. 6, pp. 1035-1039, 2014.

[20] G. E. Eperon et al., "Perovskite-perovskite tandem photovoltaics with optimized bandgaps," Science, vol. 354, pp. 861-865, 2016.

[21] G. E. Eperon et al., "Formamidinium lead trihalide: A broadly tunable perovskite for efficient planar heterojunction solar cells," Energy Environ. Sci., vol. 7, no. 3, pp. 982-988, 2014.

[22] E. L. Unger et al., "Correction: Roadmap and roadblocks for the band gap tunability of metal halide perovskites," J. Mater. Chem. A, vol. 5, no. 30, pp. 15983-15983, 2017.

[23] F. Sahli et al., "Improved optics in monolithic perovskite/silicon tandem solar cells with a nanocrystalline silicon recombination junction," $A d v$. Energy Mater, vol. 8, no. 6, 2017, Art. no. 1701609.

[24] F. Sahli et al., "Fully textured monolithic perovskite/silicon tandem solar cells with $25.2 \%$ power conversion efficiency," Nature Mater., vol. 17 , no. 9 , pp. 820-826, 2018.

[25] R. M. Swanson, "Approaching the 29\% limit efficiency of silicon solar cells," in Proc. Conf. Rec. 31st IEEE Photovolt. Spec., 2005, pp. 889-894.

[26] J. Melskens et al., "Passivating contacts for crystalline silicon solar sells: From concepts and materials to prospects," IEEE J. Photovolt., vol. 8, no. 2, pp. 373-388, 2018.

[27] F. Feldmann, M. Bivour, C. Reichel, M. Hermle, and S. W. Glunz, "Passivated rear contacts for high-efficiency n-type Si solar cells providing high interface passivation quality and excellent transport characteristics," Sol. Energy Mater. Sol. Cells, vol. 120, pp. 270-274, 2014. 
[28] D. Yan, A. Cuevas, J. Bullock, Y. Wan, and C. Samundsett, "Phosphorusdiffused polysilicon contacts for solar cells," Sol. Energy Mater. Sol. Cells, vol. 142, pp. 75-82, 2015.

[29] A. Richter et al., "n-Type Si solar cells with passivating electron contact: Identifying sources for efficiency limitations by wafer thickness and resistivity variation," Sol. Energy Mater. Sol. Cells, vol. 173, pp. 96-105, 2017.

[30] J. Cho et al., "Passivating electron-selective contacts for silicon solar cells based on an a-Si:H/TiOxstack and a low work function metal," Prog. Photovolt., Res. Appl., vol. 26, no. 10, pp. 835-845, 2018.

[31] J. Cho et al., "Performance and thermal stability of an a-Si:H/TiOx/Yb stack as an electron-selective contact in silicon heterojunction solar cells," ACS Appl. Energy Mater, vol. 2, no. 2, pp. 1393-1404, 2019.

[32] J. Bullock et al., "Efficient silicon solar cells with dopant-free asymmetric heterocontacts," Nature Energy, vol. 1, no. 3, 2016, Art. no. 15031.

[33] S. Y. Herasimenka, W. J. Dauksher, and S. G. Bowden, " $>750 \mathrm{mV}$ open circuit voltage measured on $50 \mu \mathrm{m}$ thick silicon heterojunction solar cell," Appl. Phys. Lett., vol. 103, no. 5, 2013, Art. no. 053511.

[34] M. Taguchi et al., "24.7\% record efficiency HIT solar cell on thin silicon wafer," IEEE J. Photovolt., vol. 4, no. 1, pp. 96-99, Jan. 2014.

[35] A. Descoeudres et al., "Improved amorphous/crystalline silicon interface passivation by hydrogen plasma treatment," Appl. Phys. Lett., vol. 99, no. 12,2011 , Art. no. 123506.

[36] J. Ge, Z. P. Ling, J. Wong, T. Mueller, and A. G. Aberle, "Optimisation of intrinsic a-Si:H passivation layers in crystalline-amorphous silicon heterojunction solar cells," Energy Procedia, vol. 15, pp. 107-117, 2012.

[37] K. Masuko et al., "Achievement of more than 25\% conversion efficiency with crystalline silicon heterojunction solar cell," IEEE J. Photovolt., vol. 4, no. 6, pp. 1433-1435, Nov. 2014.

[38] P. Sheng, A. N. Bloch, and R. S. Stepleman, "Wavelength-selective absorption enhancement in thin-film solar cells," Appl. Phys. Lett., vol. 43, no. 6 , pp. 579-581, 1983.

[39] C. Heine and R. H. Morf, "Submicrometer gratings for solar energy applications," Appl. Opt., vol. 34, no. 14, pp. 2476-2482, 1995.

[40] Z. Yu, A. Raman, and S. Fan, "Fundamental limit of light trapping in grating structures," Opt. Express, vol. 18, no. S3, pp. A366-A380, 2010.

[41] M. S. Branham et al., "Silicon solar cells: $15.7 \%$ efficient $10-\mu$ m-thick crystalline silicon solar cells using periodic nanostructures," Adv. Mater., vol. 27, no. 13, pp. 2268-2268, 2015.

[42] A. Mavrokefalos, S. E. Han, S. Yerci, M. S. Branham, and G. Chen, "Efficient light trapping in inverted nanopyramid thin crystalline silicon membranes for solar cell applications," Nano Lett., vol. 12, no. 6, pp. 2792-2796, 2012.

[43] M. Peters, M. Rüdiger, H. Hauser, M. Hermle, and B. Bläsi, "Diffractive gratings for crystalline silicon solar cells-optimum parameters and loss mechanisms," Prog. Photovolt., Res. Appl., vol. 20, no. 7, pp. 862-873, 2011.

[44] C. Trompoukis, O. E. Daif, V. Depauw, I. Gordon, and J. Poortmans, "Photonic assisted light trapping integrated in ultrathin crystalline silicon solar cells by nanoimprint lithography," Appl. Phys. Lett., vol. 101, no. 10, 2012, Art. no. 103901.

[45] C. Trompoukis et al., "Photonic nanostructures for advanced light trapping in thin crystalline silicon solar cells," Physica Status Solidi A, vol. 212, no. 1, pp. 140-155, 2014.

[46] V. Depauw et al., "Micrometer-thin crystalline-silicon solar cells integrating numerically optimized 2-D photonic crystals," IEEE J. Photovolt., vol. 4, no. 1, pp. 215-223, Jan. 2014.

[47] S. Koynov, M. S. Brandt, and M. Stutzmann, "Black nonreflecting silicon surfaces for solar cells," Appl. Phys. Lett., vol. 88, no. 20, 2006, Art. no. 203107.

[48] J. Oh, H.-C. Yuan, and H. M. Branz, "An 18.2\%-efficient black-silicon solar cell achieved through control of carrier recombination in nanostructures," Nature Nanotechnol., vol. 7, no. 11, pp. 743-748, 2012.
[49] H. Savin et al., "Black silicon solar cells with interdigitated backcontacts achieve $22.1 \%$ efficiency," Nature Nanotechnol., vol. 10, no. 7 , pp. 624-628, 2015.

[50] G. Yang et al., "High-efficiency black IBC c-Si solar cells with poly-Si as carrier-selective passivating contacts," Sol. Energy Mater. Sol. Cells, vol. 186, pp. 9-13, 2018.

[51] A. Ingenito, O. Isabella, and M. Zeman, "Nano-cones on micro-pyramids: Modulated surface textures for maximal spectral response and highefficiency solar cells," Prog. Photovolt., Res. Appl., vol. 23, no. 11, pp. 1649-1659, 2015.

[52] V. Depauw et al., "Sunlight-thin nanophotonic monocrystalline silicon solar cells," Nano Futures, vol. 1, no. 2, 2017, Art. no. 021001.

[53] P. Spinelli, M. Verschuuren, and A. Polman, "Broadband omnidirectional antireflection coating based on subwavelength surface Mie resonators," Nature Commun., vol. 3, no. 1, 2012, Art. no. 692.

[54] M. V. Lare, F. Lenzmann, and A. Polman, "Dielectric back scattering patterns for light trapping in thin-film Si solar cells," Opt. Express, vol. 21, no. 18, pp. 20738-20746, 2013.

[55] P. Spinelli, B. K. Newman, and A. Polman, "Photovoltaics: Light-trapping in crystalline silicon and thin-film solar cells by nanostructured optical coatings," in Nanotechnology for Energy Sustainability, B. Raj, M. V. de Voorde, and Y. Mahajan, Eds., Weinheim, Germany: Wiley, 2017, pp. $163-180$.

[56] V. Neder, S. L. Luxembourg, and A. Polman, "Efficient colored silicon solar modules using integrated resonant dielectric nanoscatterers," Appl. Phys. Lett., vol. 111, no. 7, 2017, Art. no. 073902.

[57] A. Bozzola, M. Liscidini, and L. C. Andreani, "Photonic light-trapping versus Lambertian limits in thin film silicon solar cells with 1D and 2D periodic patterns," Opt. Express, vol. 20, no. S2, pp. A224-A244, 2012.

[58] A. Razzaq et al., "Periodic inverse nanopyramid gratings for light management in silicon heterojunction devices and comparison with random pyramid texturing," Sol. Energy Mater. Sol. Cells, vol. 206, 2020, Art. no. 110263.

[59] L. C. Andreani, A. Bozzola, P. Kowalczewski, and M. Liscidini, "Photonic light trapping and electrical transport in thin-film silicon solar cells," Sol. Energy Mater. Sol. Cells, vol. 135, pp. 78-92, 2015.

[60] R. Cariou et al., "III-V-on-silicon solar cells reaching 33\% photoconversion efficiency in two-terminal configuration," Nature Energy, vol. 3, no. 6, pp. 326-333, 2018.

[61] H. Schift, "Nanoimprint lithography: An old story in modern times? A review," J. Vac. Sci. Technol. B, Microelectron. Nanometer Struct., vol. 26, no. 2, pp. 458-480, 2008.

[62] C. S. Schuster et al., "Dual gratings for enhanced light trapping in thin-film solar cells by a layer-transfer technique," Opt. Express, vol. 21, no. S3, pp. A433-A439, 2013.

[63] H. S. Radhakrishnan et al., "Module-level cell processing of silicon heterojunction interdigitated back-contacted (SHJ-IBC) solar cells with efficiencies above 22\%: Towards all-dry processing," in Proc. IEEE 43rd Photovolt. Spec. Conf., 2016, pp. 1182-1187.

[64] M. Xu et al., "Silicon heterojunction interdigitated back-contact solar cells bonded to glass with efficiency $>21 \%$," Sol. Energy Mater. Sol. Cells, vol. 165, pp. 82-87, 2017.

[65] H. S. Radhakrishnan et al., "A novel silicon heterojunction IBC process flow using partial etching of doped a-Si:H to switch from hole contact to electron contact in situ with efficiencies close to 23\%," Prog. Photovolt., Res. Appl., vol. 27, no. 11, pp. 959-970, 2019.

[66] J. P. Mailoa et al., "Energy-yield prediction for II-VI-based thin-film tandem solar cells," Energy Environ. Sci., vol. 9, no. 8, pp. 2644-2653, 2016.

[67] M. T. Hörantner and H. J. Snaith, "Predicting and optimising the energy yield of perovskite-on-silicon tandem solar cells under real world conditions," Energy Environ. Sci., vol. 10, no. 9, pp. 1983-1993, 2017.

[68] E. Özkol et al., "Effective passivation of black silicon surfaces via plasma-enhanced chemical vapor deposition grown conformal hydrogenated amorphous silicon layer," Physica Status Solidi, Rapid Res. Lett., vol. 14 , no. 1, 2019, Art. no. 1900087. 